Annuaire suisse de politique de développement

13 | 1994

Annuaire Suisse - Tiers Monde 1994

\title{
VII. Aide aux pays de l'Estn
}

\section{(2) OpenEdition}

1 Journals

Édition électronique

URL : http://journals.openedition.org/aspd/1037

DOI : $10.4000 /$ aspd. 1037

ISSN : 1663-9669

Éditeur

Institut de hautes études internationales et du développement

\section{Édition imprimée}

Date de publication : 1 janvier 1994

Pagination : 155-158

ISSN : 1660-5934

\section{Référence électronique}

"VII. Aide aux pays de l'Estn», Annuaire suisse de politique de développement [En ligne], 13| 1994, mis en ligne le 19 décembre 2012, consulté le 08 septembre 2020. URL : http://journals.openedition.org/ aspd/1037 ; DOI : https://doi.org/10.4000/aspd.1037 


\section{Aide aux pays de l'Est}

La Suisse a réagi très rapidement au processus de transformation qui s'est déclenché dans les pays de l'ancien bloc de l'Est et qui a acquis, depuis la chute du murde Berlin le 9 novembre 1989, une dynamiqueetune dimension impressionnantes. Elle a en effet mis à disposition deux crédits-cadres d'un total de 1'650 millions de francs pour financer l'aide aux pays de l'Est. Celle-ci comprend pour l'essentiel une aide financière, qui englobe trois quarts environ des crédits-cadres consentis, et la coopération technique (un quart des crédits). Un arrêté fédéral, dont la portée doit s'étendre sur dix ans et dont l'adoption est prévue en 1994, constituera la base légale de cette aide. Par "pays de l'Est", nous entendons ici les pays de l'Europe de l'Est et les pays de la Communauté des Etats indépendants.

\section{Crédits-cadres}

Après un bref débat mené au mois de juin 1990, le Parlement a mis à disposition un premier crédit pour l'aide aux pays de l'Est: premier crédit-cadre de 250 millions de francs en faveur des pays d'Europe de l'Est. II fut affecté à la coopération avec la Pologne, l'ancienne Tchécoslovaquie et la Hongrie.

En janvier 1992, ce premier crédit fut suivi d'un deuxième crédit-cadre de plus de 800 millions de francs, qui a permis d'élargir l'aide aux pays suivants: Albanie, Bulgarie, Etats baltes (Estonie, Lettonie, Lituanie), ainsi que Croatie, Slovénie et Roumanie. II a également servi à financer des projets ponctuels dans les pays de la Communauté des Etats indépendants (CEI).

En mars 1993, ce deuxième crédit-cadre a été porté à 1'400 millions de francs et ouvert à tous les pays de la CEI et à la Géorgie.

Forme de l'aide et compétences

La coopération prend le plus souvent la forme d'une aide financière. Les trois quarts des deux crédits-cadres sont consacrés à ce type d'aide, soit quelque 1'250 millions 
de francs. L'aide financière relève de la compétence du "Service de mesures économiques pour l'Europe centrale et orientale", récemment créé au sein du Département fédéral de l'économie publique. Un quart de l'aide est fournie sous forme de coopération technique (400 millions de francs environ); sa gestion incombe au "Bureau de coopération pour l'Europe de l'Est" du Département fédéral des affaires étrangères.

Les pays de l'Est bénéficient d'autres mesures de soutien sous forme d'aide humanitaire. Pour répondre à la hausse massive des besoins rencontrés dans ces pays, l'aide humanitaire de la Confédération s'est nettement accrue ces dernières années. Ses dépenses ont atteint 1,1 million de francs en 1990, 8,2 millions déjà en 1991 et 47,6 millions de francs en 1992, dont 38,6 millions étaient destinés aux victimes du conflit de l'ancienne Yougoslavie. L'aide humanitaire est financée par un crédit séparé couvrant le monde entier et gérée par la Direction de la coopération au développement et de l'aide humanitaire, au sein du DFAE.

L'aide à la balance des paiements est une autre mesure d'aide aux pays de l'Est. L'arrêté de garantie" de 1975 constitue sa base légale. Concrètement, l'aide à la balance des paiements comprend des crédits, portant intérêts, d'une durée maximale de sept ans, accordés par la Banque nationale suisse et garantis par la Confédération. L'aide à la balance des paiements est un moyen d'aide rapide. Elle n'est pas liée et sert à payer des importations et à augmenter les réserves en devises. Seuls les pays dont la politique économique répond aux critères du FMI peuvent bénéficier de cette aide. En 1991, la Hongrie, l'ex-Tchécoslovaquie, la Roumanie et la Bulgarie ont obtenu une aide à la balance des paiements d'un total de 200 millions de francs. En 1992, la Suisse a promis de l'Aide à la Roumanie, à la Bulgarie et aux Etats baltes. En juillet 1993 pourtant, seul le contrat avec la Roumanie a été signé, qui porte sur un montant de 7,2 millions de francs.

Dans le cadre de son aide aux pays de l'Est, la Suisse participe aussi à des actions internationales. Elle est notamment membre de la Banque européenne de reconstruction et de développement (BERD), du Fonds monétaire international (FMI) et de la Banque mondiale, du G-24 (groupe des 24 pays industrialisés les plus riches du monde) et del'Organisation de coopération et de développementéconomique (OCDE); elle participe aussi à leurs programmes d'aide.

\section{Principes de la coopération avec les pays de l'Est}

L'aide suisse aux pays de l'Est a pour but de soutenir les processus de réforme engagés dans ces pays en faveur d'une démocratie ouverte et de l'économie de marché. Au travers de son aide, la Suisse poursuit avant tout les objectifs suivants (1):

- La coopération politique pour soutenir le processus de démocratisation.

- La coopération économique, dans le cadre d'un développement économique respectueux de l'environnement, vise à réduire la différence de niveau de vie entre l'Est et l'Ouest.

- La coopération juridique doit resserrer les liens entre Etats et favoriser le règlement pacifique des différends. 
- La Suisse désire par ailleurs développer les contacts sociaux, scientifiques et culturels.

- Elle veut contribuer à la solution de problèmes de dimension internationale (pollution de l'environnement, politique de sécurité).

La Suisse souhaite ainsi répondre aux vœux émis par les pays engagés dans les réformes. La situation pouvant évoluer très rapidement, cette coopération exige beaucoup de flexibilité. La Suisse concentre son aide sur des domaines et des projets dont elle peut attendre le plus grand rayonnement possible dans le sens des objectifs cités ci-dessus. Ces programmes doivent être considérés comme partie intégrante des mesures de soutien occidentales. Comme pour la coopération avec les pays en développement, l'objectif premier de l'appui aux pays de l'Est est de les aider à s'aider eux-mêmes, de promouvoir l'initiative privée et d'éveiller le sens des responsabilités. En règle générale, les autorités fédérales ne se chargent pas de la réalisation des projets, mais les confient à des tiers sous forme de mandats.

\section{Principaux points de la coopération}

La coopération technique et l'aide financière sont les principaux domaines de l'aide aux pays de l'Est. Dans le cadre de la coopération technique, la Suisse soutient des projets dans les pays bénéficiaires en mettant à disposition du personnel qualifié chargé de transmettre des connaissances dans des domaines aussi variés que la politique et l'organisation de l'Etat, l'économie, l'agriculture et l'approvisionnement, la santé, le social, les sciences et la formation, l'environnement et l'énergie, la culture. Dans le cadre du soutien aux pays de l'Est, la Confédération a créé un nouvel instrument qui est l'aide de bon voisinage. Elle vise à atténuer les graves conséquences sociales liées aux programmes de réformes et rendre ainsi moins pénibles les privations que doit endurer une bonne partie de la population. La livraison par la Croix-Rouge suisse de produits pharmaceutiques de première nécessité à l'Estonie et à la Lettonie en est un exemple.

L'aide financière est accordée là où il existe des besoins de produits suisses qui ne peuvent être financés commercialement. II s'agit avant tout de projets dans les domaines de la santé, de l'infrastructure et de l'environnement, qui sont appuyés par des contributions financières. Les garanties de crédit servent à financer des livraisons de marchandises et des services à des eritreprises de production et pour des projets d'infrastructure. La situation économique dans nombre de pays de l'Est représente un risque trop élevée pour qu'ils puissent bénéficier de la garantie contre les risques à l'exportation (GRE), mais le besoin en importations est très grand. Ces risques peuvent être couverts par des garanties de crédit prélevées sur les créditscadres. L'aide financière est accordée à condition qu'elle serve à financer des "besoins prioritaires". La coopération technique et l'aide financière sont complémentaires et elles sont souvent combinées dans la pratique.

Les prestations fournies en 1992 par la Confédération en faveur des pays de l'Est sont détaillées par pays dans une publication sur l'aide suisse aux pays en développement. Selon ces données, la Confédération a consacré un total de 184 
millions de francs à l'aide aux pays de l'Est, dont 169 millions à titre d'aide bilatérale et 25 millions à titre d'aide multilatérale. Ces données diffèrent de celles fournies par le Bureau de coopération pour l'Europe de l'Est selon lesquelles l'aide de la Confédération à ces pays s'est montée à 159,9 millions de francs en 1992, soit 47,6 millions de francs pour l'aide humanitaire, 105,1 millions de francs pour l'aide financière et la coopération technique et 7,2 millions de francs pour l'aide à la balance des paiements.

Arrêté fédéral concernant la coopération avec les Etats de l'Est

L'aide aux pays de l'Est se fonde simplement sur un arrêté fédéral pris conformément à l'article 8 de la loi sur l'organisation de l'administration et elle est régie par I'"ordonnance concernant les mesures pour renforcer la coopération avec les Etats d'Europe orientale" du 6 mai 1992. Etant donné que la Constitution confère au Conseil fédéral la compétence de gérer les affaires extérieures et que le Parlement est compétent en matière budgétaire, la Suisse a pu réagir rapidement face aux réformes engagées dans les pays de l'Est et les soutenir par une aide substantielle. L'expérience a montré que le processus de réforme exige du temps et qu'il convient donc de mettre en place une aide à moyen et à long terme. La coopération avec les pays de l'Est deviendra probablement partie intégrante des relations extérieures de la Suisse et engendrera d'importants frais financiers, administratifs et de personnel. Un arrêté fédéral concernant la coopération avec les Etats de l'Est est en préparation.

\section{Note}

1. Ces objectifs sont décrits dans La Suisse, l'Europe de l'Est et la CEI - Documentation concernant le programme de soutien de la Confédération aux Etats d'Europe orientale et aux Etats de la CEI (Etat fin juillet 1993), édité par le Bureau de coopération pour l'Europe de l'Est (DFAE) et par le Service des mesures économiques pour l'Europe centrale et orientale (OFAEE).

\section{Sources}

Bureau de coopération pour l'Europe de l'Est/DFAE

- La Suisse et les pays d'Europe centrale et orientale, Documentation sur le programme de soutien de la Confédération aux pays d'Europe centrale et orientale, Berne, mars 1993

- Documentation sur les projets de la coopération technique avec les pays d'Europe orientale, Berne, août 1993

Ordonnance concernant les mesures pour renforcer la coopération avec les pays d'Europe orientale, 6 mai 1992

DFAE, avant-projet d'arrêté fédéral concernant la coopération avec les Etats d'Europe de l'Est (procédure de consultation: juin à septembre 1993) et commentaire explicatif, Berne, juin 1993 